\title{
Retrieval Technology by Example During Product Development of Auto Parts
}

\author{
Jun Chen ${ }^{1,2}$ \\ ${ }^{1}$ Mechanical and Electronic Engineering College \\ Hubei Polytechnic University \\ 16, Guilin North Road, 435003, Huangshi, P. R. China \\ cjund@163.com
}

\author{
He Huang ${ }^{2}$, Ting $\mathrm{Wu}^{2}$, Bin Duan ${ }^{2}$ \\ ${ }^{2}$ School of Mechanical and Electronic Engineering \\ Wuhan University of Technology \\ 122 Luoshi Road, 430070, Wuhan, Hubei Province, \\ China \\ flying-0415@163.com,393840532@qq.com, \\ duanbin1101@126.com
}

\begin{abstract}
In this paper, aiming at the characteristics of auto parts enterprises' product development, the object-oriented case knowledge expression to build auto parts product development database is used, the nearest neighbor algorithm is as similarity algorithm of case retrieval, the TCP/IP network technology is as the main network transmission protocol, $\mathrm{C} / \mathrm{S}$ is as the application system to build the case retrieval system, and EPS steering gear is as an example.
\end{abstract}

Keywords-auto parts;product development;example; retrieval

\section{INTRODUCTION}

The product development of inheritance and adaptability which rely on the original research is that use the existing technology to develop, revise and innovate on the basis of example. During process, due to the massive application examples and data structure and query mechanism problems ,it is difficult to find the proper examples. This article attempts to solve the practical engineering problems from the establishment and use of instance database.

\section{THE ESTABLISHMENT OF INSTANCE DATABASE DURING PRODUCT DEVELOPMENT OF AUTO PARTS}

\section{A. The object-oriented case knowledge expression}

The examples can be expressed in different ways, like semantic web, database, prototype, neural network, memory network representation, object-oriented case etc[1]. Generally, the object-oriented case knowledge expression with modularized structure can satisfy the need of various kinds of knowledge and maintain the case library. Objectoriented case contains two basic elements: the description of data and method that combine them to make them structured and modular[2]. So an example includes categories, properties, decision results, conclusion and general and domain knowledge.

\section{B. The establishment of database for object-oriented case knowledge expression}

Take the gear design of EPS in an enterprise for example. During the product development,the project technology personnel will locate the closest example of requirement for project design according to the characteristic property without calculating the parameters of the product. If EPS steering gear is cylindrical helical gear, the characteristic parameters designers concern about are power input, input torque, transmission ratio, center distance that are not easily changed. The parameters are named as the basic design attribute. There are some parameters such as tooth shape, accuracy class, a predetermined working life that affect gear. They are called aided design attribute parameters which are important in gear design. So when establish the database, the basic design attribute and aided design are index entry for example.Other parameters such as the width of teeth, tooth, tooth surface hardness, material and heat treatment that those values cannot be predicted accurately before accomplishment are not involved in the case retrieval but be stored in the case base, when retrieve the case the feature values are displayed the details, then the project staffs will choose according to the need. At the same time, the example also contains the displayed attributes. The instance properties are shown in figure 1.

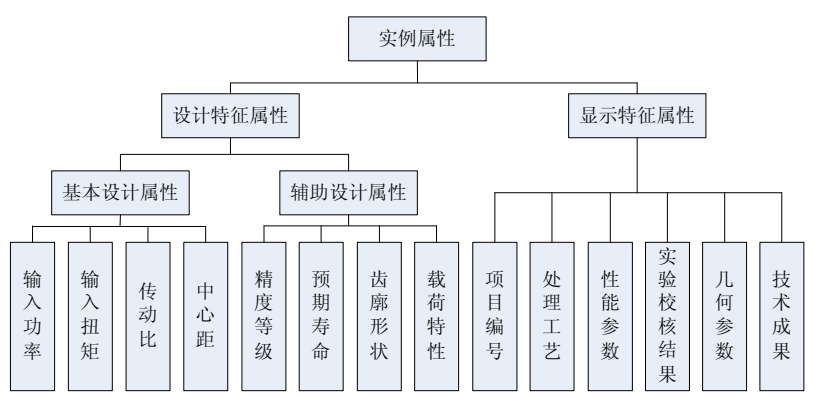

Figure 1. The case properties of EPS

The case feature attribute should be able to express complete and accurate data and information including the digital information, symbolic information, quantitative and qualitative information, accurate information and fuzzy information etc. Therefore, in order to establish the case base according to existing design examples, we should have a unified knowledge model framework to express product design process involving all kinds of knowledge that enable the designer to find and call out the most valuable knowledge and information quickly and efficiently according to the required content of different design stages and lead designers to design required product quickly[4]. 


\section{THE SIMILARITY ALGORITHM OF CASE RETRIEVAL FOR AUTO PARTS PRODUCT DEVELOPMENT}

\section{A. Feature attribute assignment}

Before the calculation of case similarity we should determine the method. Feature similarity refers to the similar degree of two examples that the range is between 0 and 1.0 represents two completely different, 1 means two fully consistent. The data types of calculation for feature similarity are numeric, text etc[5-6], namely similarity can be quantitatively represented while some are only be described. As the load characteristic, the load instability characteristics can be set into a quantitative expression , the value is 1 if vibrate, value is 0 if stable, select the valves between 0 and 1 according to the actual load. (1).

The method for numeric feature assignment is as formula

$$
p_{m n}=\frac{f_{m n}-\min f_{n}}{\max f_{n}-\min f_{n}}
$$

$p_{m n}$ represents the $\mathrm{n}$ attribute assignments of $\mathrm{m}$ instance, $f_{m n}$ represents the $\mathrm{n}$ attribute value of $\mathrm{m}$ instance, $\min f_{n}$ means the minimum value, $\operatorname{maxf}_{n}$ means the maximum value.

\section{B. The calculation of case similarity}

The common algorithm of case similarity retrieval has nearest neighbor strategy, TC similarity method, inductive reasoning strategy, knowledge guidance strategy and template retrieval strategy. This article selects the nearest neighbor algorithm.

According to the characteristics of attribute assignment algorithm, firstly calculate every attribute similarity value of various examples. For example, $p_{m n}$ is stored in the database. The user according to the requirement of design, input the similarity value $p_{n}$, then the distance between the two is:

$$
d_{m n}=\left|p_{n}-p_{m n}\right|
$$

The overall distance for example is:

$$
d_{m}=\sum d_{m n} \times w_{n}
$$
of $n$.

Among them, wn represents the attribute weight value

The similarity between design issues and examples is :

$$
\eta_{\mathrm{m}}=1-d_{m}
$$

IV. THE DESIGN AND IMPLEMENTATION OF CASE RETRIEVAL SYSTEM FOR AUTO PARTS PRODUCT DEVELOPMENT

\section{A. The design of case retrieval system for auto parts product development}

1)The structure of case retrieval system:The structure of case retrieval system for auto parts product development is as figure2.

In the system design, use TCP/IP network technology as the main network transmission protocol, use C/S (client / server) structure model for application system.

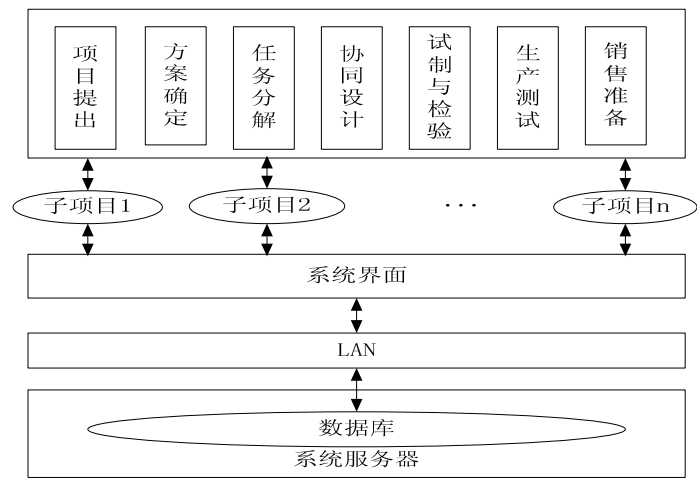

Figure 2. The structure of case retrieval system for auto parts product development

Sub-item.Sub-item is a concrete unit for collaborative innovation. Through collaborative innovation platform, technology and information are shared for resource allocation.

LAN.Select LAN because it is mainly related to the resource share of interior enterprise. Project personnel that participate in product development use C/S (client / server) mode. The business logic is achieved in the application server. The user operate the interface through the browser. System server is arranged in product development project management department and the project management department is responsible for the daily maintenance.

System interface. The system interface is a window to participate in collaborative innovation for product development project personnel. System interface provides all function buttons, for example: the landing interface, project progress, project results to the query, exchange of technology and so on.

Server. The server is the core of the system. Database is installed on the server that store related data and provide specific access mechanism. The database is the basis platform for system operation. At the same time, during the development, it produces a large number of solutions, information documents and a lot of projects, tasks and other information resources, those are stored in the database. The company's project management maintain the database to ensure security and confidentiality.

2)Database and development tools:Hardware platform is the necessary hardware environment for development and operation, including computer models and hardware configuration.The requirements for system to the computer hardware is not high, ordinary computer of $256 \mathrm{M}$ memory (or above), 20G hard drive (or more)can run smoothly.

The software platform is the software environment for system development and operation. When system develops, select mainstream Win7 ultimate system and C\# as developing language.In order to improve the stability, considering the compatibility issues, select the Microsoft Access database.

C\# has a visual development environment that can quickly complete development of system. The internal 
integrated database access channel can easily manage database.

Database uses Microsoft Access. Due to the system requirements of design, Microsoft Access can fit the demand of work with rich supported data type, convenient maintenance, low costs and not high personnel quality.

3)The conceptual design of database:Conceptual design known as the conceptual model is that according to a certain method do abstract and comprehensive treatment to data basing on the demand analysis and design the information structure not depending on a specific DBMS to meet the needs of the user application.This paper choose entity analysis methods, including entity selection, determine the contact, determine the attribute.

The first step: entity selection.This article selects the entity including the project manager, project members, administrators, project information, patent information, patent forecast result, technology sharing platform.

The second step: determine the contact. It is associations between entities. This database contains the original instance data and instance data from research process.

The third step: determine the attribute.

- $\quad$ project member attributes: ID, username, password, the involved project, way of contact. Certificate number is primary key.

- $\quad$ project manager attributes: ID, username, password, the involved project, way of contact. Certificate number is primary key.

- $\quad$ system administrator attribute: administrator number, login, password, real name.Manager ID is primary key.

- $\quad$ project information attribute: project number, item name, research object, research type, start date, technology, manpower, equipment requirements. Project number is primary key.

- patent information attribute: patent name,patent number, object, type, keyword, technical achievement, applicant, date of application, applicant, applicant contact way. Patent number is primary key.

- property of the results predicted by patent dates: number, R\&D object ,type of $R \& D$, research and development of content, technology trends. Primary key is number.

- $\quad$ property of technical shared resource: number, $R \& D$ object, type of R\&D, technological achievements. Technical shared resource is intergrated by project information and patent information,and merged Corresponding to the $R \& D$ objects, type of $R \& D$ and technological achievements from these two forms. Primary key is number. Database field attributes and relationships are shown in Figure 3.

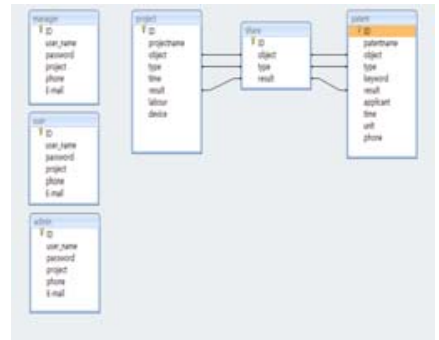

Figure 3. Database field attributes and relationships

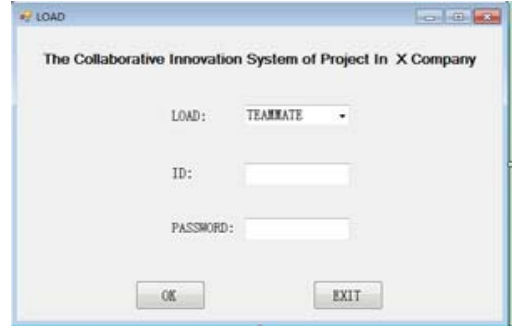

Figure 4. System login screen

\section{B. Retrieval of instances in the development of auto parts product}

On the basis of system design ideas,combining with the characteristics of the automotive parts manufacturers, This paper selects automobile steering products as the object for research and development,and uses this design in the projects of steering.

1)View of technical information:There are three types of landing:project members, project managers, system administrators, we need to enter a user name and password correctly and then enter a different interface.System login screen is shown in Figure 4.

After entering the system, choose technical shared resource and you can view all the company project examples,refer to similar technological achievements of the project and the $\mathrm{R} \& \mathrm{D}$ process.In addition, we can select patent data predicted results to see the technology trends, provide the basis for the positioning of the product development.They can help provide the basis for the position of the product development.

2)Project case retrieval:This paper takes the design of gear for example.According to design requirements, $R \& D$ and minimum similarity are selected in the interface, then enter the initial values and weights of the design attributes.The system can automatically retrieves all instances meet similarity. The detailed information of project examples provide parameters and technical means,and reflects the system's function of sharing technical resources.As shown in figure 5. Choose a project and you can get the detailed information of it,as shown in figure 6 . 


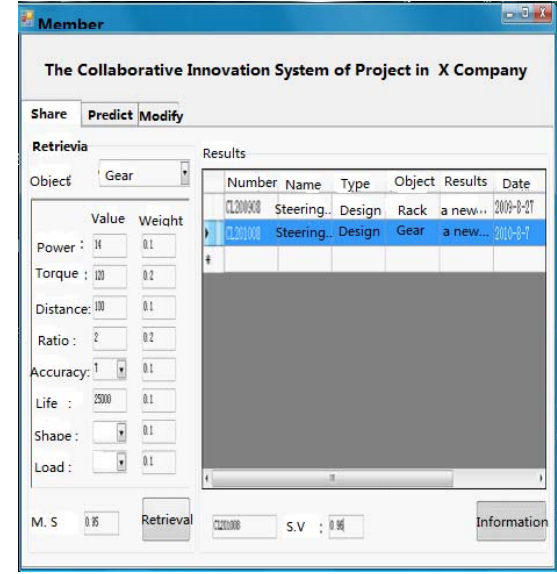

Figure 5. Sample of retrieval interface

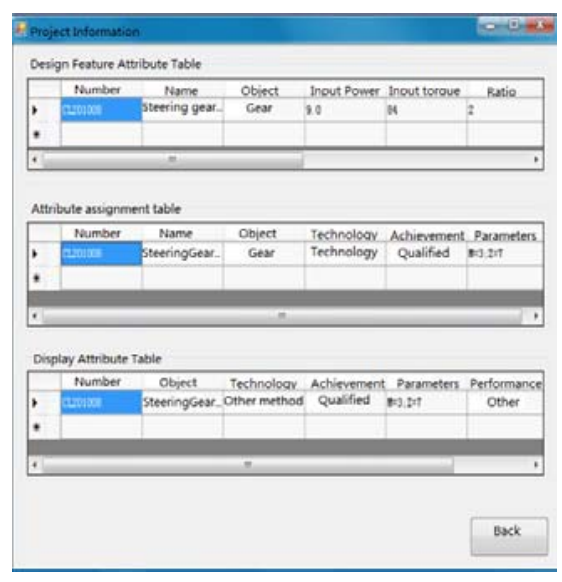

Figure 6. Examples of search results

3)The implementation multi-project collaborative innovation management system :Project Leader get the detailed information from data output interface,and the information is callable.Use similarity algorithm to determine the best similar instance and to identify the characteristic properties that does not match with the design requirements. Then make some adjustment whit the appropriate methods. After all test the new design results to see if all meet the design constraints.If all the constraints are satisfied, then the design is complete; if there are still some shortage,make a further modification until all the requirements are satisfied with the constraints.

There are three main method of modifying instance: modify instances by the combination of instances, knowledge-based modification and manually modify. After modification the result can be formed a new instance and added to the case library with some strategies. It can be used in the development process of subsequent products.

\section{V.CONCLUSION}

With the research of the establishment of the instance database,the similarity algorithm in instance retrieval, the design and implementation of instance retrieval system,it provides a solution in using the instances in auto parts product development.

\section{REFERENCES}

[1] Haitao Ren.Case-based reasoning and its use in agricultural expert system[D].Shanxi:Shanxi University,2004

[2] Haitao Ren, Ru Li etc. Realization Approach Research on CBR Agricultural Expert System, In Proceeding of the Second Intenrational Symposium on Intelligent Inofmration Technology in Agrieulture Agriculture Perss of China, 2004.

[3] Yanni Meng.Instance-based gear design application technology research[D],Xi'an:Northwestern Polytechnical University,2006

[4] Weiqing Ling,Wenping Zhao, Youbai Xie. The method and implementation of accessing to knowledge in instance-based product design[J].Computer Aided Design and Computer Graphics, 2002,14(11):1014-1018.

[5] Huiqing Li. Modeling and Application of mechanical product design knowledge[D]. Zhejiang:Zhejiang university,2011

[6] Feng Qi.Product design information reusability and research of product design resource management key technology[D]. Zhejiang:Zhejiang university,2004. 